\title{
Differential gene expression analysis and network construction of recurrent cardiovascular events
}

\author{
JIANGQUAN LIAO ${ }^{1,2^{*}}$, ZHONG CHEN $^{1,2^{*}}$, QINGHONG HE ${ }^{1 *}$, YONGMEI LIU ${ }^{1}$ and JIE WANG ${ }^{1}$ \\ ${ }^{1}$ Department of Cardiology, Guang'anmen Hospital, China Academy of Chinese Medical Sciences, Beijing 100053; \\ ${ }^{2}$ Graduate School, Beijing University of Chinese Medicine, Beijing 100059, P.R. China
}

Received January 10, 2015; Accepted November 2, 2015

DOI: $10.3892 / \mathrm{mmr} .2015 .4707$

\begin{abstract}
Recurrent cardiovascular events are vital to the prevention and treatment strategies in patients who have experienced primary cardiovascular events. However, the susceptibility of recurrent cardiovascular events varies among patients. Personalized treatment and prognosis prediction are urged. Microarray profiling of samples from patients with acute myocardial infarction (AMI), with or without recurrent cardiovascular events, were obtained from the Gene Expression Omnibus database. Bioinformatics analysis, including Gene Oncology (GO) and Kyoto Encyclopedia of Genes and Genomes (KEGG), were used to identify genes and pathways specifically associated with recurrent cardiovascular events. A protein-protein interaction (PPI) network was constructed and visualized. A total of 1,329 genes were differentially expressed in the two group samples. Among them, 1,023 differentially expressed genes (DEGs; 76.98\%) were upregulated in the recurrent cardiovascular events group and 306 DEGs (23.02\%) were downregulated. Significantly enriched GO terms for molecular functions were nucleotide binding and nucleic acid binding, for biological processes were signal transduction and regulation of transcription (DNA-dependent), and for cellular component were cytoplasm and nucleus. The most significant pathway in our KEGG analysis was Pathways in cancer $(\mathrm{P}=0.000336681)$, and regulation of actin cytoskeleton was also significantly enriched $(\mathrm{P}=0.00165229)$. In the PPI network, the significant hub nodes were GNG4, MAPK8, PIK3R2, EP300, CREB1 and PIK3CB. The present study demonstrated the underlying molecular differences between patients with AMI,
\end{abstract}

Correspondence to: Professor Jie Wang, Department of Cardiology, Guang'anmen Hospital, China Academy of Chinese Medical Sciences, 5 Beixiange, Xicheng, Beijing 100053, P.R. China

E-mail: wangjie0103@126.com

*Contributed equally

Key words: recurrent cardiovascular events, microarray profile, differential expressed genes, protein-protein interaction network, bioinformatics with and without recurrent cardiovascular events, including DEGs, their biological function, signaling pathways and key genes in the PPI network. With the use of bioinformatics and genomics these findings can be used to investigate the pathological mechanism, and improve the prevention and treatment of recurrent cardiovascular events.

\section{Introduction}

With the significant advances in medication, reperfusion therapy, cardiac rehabilitation and organ transplantation, cardiovascular disease remains one of the major causes of mortality worldwide (1). Evaluation of cardiovascular disease based on risk factors is important in the clinical prevention and treatment of cardiovascular disease, which may alter the risk stratification and guide the treatment and prognosis $(2,3)$. More and more indexes are included in the risk stratification as clinical and experimental research develops, including brain natriuretic peptide, $\mathrm{C}$ reactive protein and blood homocysteine. However, the prediction of cardiovascular disease is not so satisfying (4), particularly in personalized prevention and treatment. Sensitivity of risk factors varies in different individuals, and clinical doctors must be aware of this and objective to the current risk factors and stratification (5). More superior and systematic algorithms for stratification remain to be elucidated (6).

The evaluation and stratification of cardiovascular diseases depend more on primary cardiovascular events, which elevate the stratification and enhance the treatment once they occur. However, recurrent cardiovascular events are also vital, which indicate that the current intervention is not marked enough to prevent disease progression. Although patients receive standard treatment based on the risk factors stratification, recurrent cardiovascular events still occur, which indicates that certain individuals are more prone to recurrent cardiovascular events. These patients may require more aggressive therapies, involving susceptibility screening and personalized treatment (7). With the development and application of clinical genomics technology and bioinformatics, novel biomarkers are used in the diagnosis and prognosis of cardiovascular disease $(8,9)$. Previous research revealed that the expression of different genes varies in different stages of cardiovascular diseases, and these genes are involved in the pathological process, and may even predict 
the cardiovascular events (10). With the help of genomics and bioinformatics, patient susceptibility to recurrent cardiovascular events may be screened out, and personalized treatment can be made. This may reduce the recurrence of cardiovascular events and improve the prognosis. The present study used genomics and bioinformatics technology, and associated software to analyze the differentially expressed genes (DEGs) associated with recurrent cardiovascular events. The present study also aimed to identify the key genes in the pathological process and provide alternative guidance in the preventions, and personalized treatment of recurrent cardiovascular events.

\section{Materials and methods}

Microarray data and clinical characteristics. The microarray dataset, GSE48060 with GPL570 [HG-U133_Plus_2] Affymetrix Human Genome U133 Plus 2.0 Array platform, was obtained from the Gene Expression Omnibus (GEO) database (11). The data samples were peripheral blood samples collected from patients with acute myocardial infarction (AMI) $48 \mathrm{~h}$ within the primary AMI. All 27 samples were divided into two groups, according to the recurrence of cardiovascular events in the 18 month follow-up. A total of five patients exhibited recurrent cardiovascular events and 22 did not. The definition of recurrent cardiovascular events is recurrent myocardial infarction, re-vascularization, evidence of restenosis, hospitalization for unstable angina or heart failure, cardiovascular mortality, stroke or transient ischemic attack, or amputation due to peripheral vascular disease.

Raw data processing. All 27 sample files were downloaded from the GEO database and were reanalyzed using $\mathrm{R}$ software (version 3.1.1; http:// www.r-project.org/). The Affy package was applied to read the probe set data from the CEL files (12). Robust Multiarray Averaging was used to normalize the original data. Following standardization, a total of 54,675 probe set IDs' expression levels in different samples were obtained.

Screening and annotation of the DEGs. The limma package in the R software was used to compare the expression levels of the probe sets between the two groups (13). The threshold was set as $\mathrm{P}<0.05$ or a fold change $>1.5$. The annotate package was used to annotate the DEGs.

Enrichment analysis of DEGs. GeneCodis online tools (http://genecodis2.dacya.ucm.es/) were used to annotate and analyze the DEGs $(14,15)$. The annotation and analysis were predominantly focussed on the molecular function, the biological process and the cellular component of Gene Ontology (GO) enrichment and Kyoto Encyclopedia of Genes and Genomes (KEGG) pathway analysis. The threshold was set as $\mathrm{P}<0.05$.

Protein-protein interaction (PPI) network analysis. Cytoscape (version 3.1.1; The Cytoscape Consortium, San Diego, CA, USA) and reactome plugin were applied to analyze the DEGs (16), and to construct and visualize the PPI network.
Further analysis of the key nodes in the PPI network were processed.

\section{Results}

Clinical characteristics of the two group samples. According to Suresh et al (11), the clinical characteristics between the recurrence and no recurrence groups, including age, sex, body mass index, cardiovascular risk factor score, lipid profile and severity of AMI, were similar with the exception of the usage of beta-blockers. The clinical characteristic details are listed in Table I.

Recurrent cardiovascular event-associated DEGs. By comparing the two group samples of with or without recurrent cardiovascular events in the 18 month follow-up following primary AMI, 1,329 genes (2.43\% of total probe set) were identified to be differentially expressed and annotatable. A total of 1,023 DEGs (76.98\%) were upregulated and 306 DEGs (23.02\%) were downregulated in the recurrent cardiovascular events group. The top 10 markedly up or downregulated genes with a fold change $>1.5$ are listed in Tables II and III, respectively.

Significant $\mathrm{GO}$ enrichment. To gain insights into the biological roles of the DEGs in recurrent cardiovascular events, a GO categories enrichment analysis was performed using GeneCoDis. GO categories are predominantly in three groups: Biological process, cellular component and molecular function. The significantly enriched GO terms for molecular functions were nucleotide binding (GO:0000166, $\mathrm{P}=8.24^{-19}$ ) and nucleic acid binding (GO:0003676, $\mathrm{P}=1.94^{-07}$ ), for biological processes were signal transduction (GO:0007165, $\mathrm{P}=5.26^{-08}$ ) and regulation of transcription, DNA-dependent (GO:0006355, $\mathrm{P}=9.19^{-06}$ ), and for cellular component were cytoplasm $\left(\mathrm{GO}: 0005737, \mathrm{P}=8.98^{-25}\right)$ and nucleus (GO:0005634, $\mathrm{P}=1.91^{-23}$; Fig. 1).

Significant pathways. KEGG pathway enrichment analysis was performed to further evaluate the biological significance of the DEGs. The most significant pathway in our KEGG analysis was Pathways in cancer $(\mathrm{P}=0.000336681)$. Furthermore, Melanoma $(\mathrm{P}=0.000336681)$ and Regulation of actin cytoskeleton $(\mathrm{P}=0.00165229)$ were revealed to be highly enriched. The top 15 enriched KEGG pathways of the DEGs are listed in Table IV.

PPI network construction and visualization. By analyzing the identified 1,329 DEGs using Cytoscape and the reactome plugin, 330 genes (node) and 796 gene-gene interactions (edge) were identified. The result was visualized in Cytoscape and the majority of the nodes were located within one network. To modify the PPI network, the sizes of the nodes were set according to their interaction density with the other nodes. The node color of the upregulated DEGs were made red and those downregulated were made blue (Fig. 2). The more that one gene interacts with the other genes, the larger the node was and the more central this gene occurs within the network. The genes, GNG4, MAPK8 and PIK3R2 were the three predominantly upregulated genes, while EP300, CREB1 and PIK3CB were the predominantly downregulated genes in the PPI network. The details of the nodes are listed in Table V. 
Table I. Baseline clinical characteristics of AMI patients with or without recurrent events following primary AMI, who underwent whole-genome blood gene expression microarray analysis.

\begin{tabular}{|c|c|c|c|}
\hline Variable & Event $(\mathrm{n}=5)$ & No event $(n=22)$ & P-value \\
\hline Age, years & $51(41-53)$ & $56.5(48-65)$ & 0.110 \\
\hline Gender, male, n (\%) & $3(60)$ & $13(59)$ & 0.972 \\
\hline Body mass index, $\mathrm{kg} / \mathrm{m}^{2}$ & $36.5(25.0-46.5)$ & $31.5(22.9-48.4)$ & 0.140 \\
\hline Cardiovascular risk factor score & $5(4-6)$ & $4(1-6)$ & 0.266 \\
\hline \multicolumn{4}{|l|}{ Cardiovascular history, n (\%) } \\
\hline Arterial hypertension, n (\%) & $5(100)$ & $13(59)$ & 0.080 \\
\hline Smoking, n (\%) & $2(40)$ & $16(73)$ & 0.161 \\
\hline Diabetes mellitus, n (\%) & $0(0)$ & $3(14)$ & 0.381 \\
\hline Family history of coronary artery disease, $\mathrm{n}(\%)$ & $5(100)$ & $12(55)$ & 0.057 \\
\hline \multicolumn{4}{|l|}{ Lipid profile, $\mathrm{mg} / \mathrm{dl}$} \\
\hline Total cholesterol, mg/dl & $128(110-219)$ & $191(126-325$ & 0.190 \\
\hline Low density lipoprotein cholesterol, mg/dl & $65(41-152)$ & $115(70-254)$ & 0.169 \\
\hline HDL cholesterol, mg/dl & $31(25-47)$ & $38(25-72)$ & 0.165 \\
\hline \multicolumn{4}{|l|}{ Medication } \\
\hline Statin therapy, n (\%) & $3(60)$ & $7(33)$ & 0.271 \\
\hline Aspirin, n (\%) & $4(80)$ & $12(55)$ & 0.296 \\
\hline ACE inhibitor, n (\%) & $1(20)$ & $4(19)$ & 0.961 \\
\hline Beta blocker, n (\%) & $4(80)$ & $5(22)$ & 0.014 \\
\hline \multicolumn{4}{|l|}{ Severity of AMI } \\
\hline Ejection fraction, $\%$ & $55(43-65)$ & $57(35-71)$ & 0.240 \\
\hline Troponin, ng/ml & $2.24(0.11-9.51)$ & $0.47(0.04-16.43)$ & 0.142 \\
\hline STEMI, n (\%) & $2(40)$ & $7(32)$ & 0.726 \\
\hline
\end{tabular}

AMI, acute myocardial infarction.

Table II. Top 10 upregulated genes.

\begin{tabular}{|c|c|c|c|c|c|}
\hline \multirow[b]{2}{*}{ Gene } & \multirow[b]{2}{*}{ Fold change } & \multicolumn{2}{|c|}{ Mean of intensity } & \multirow[b]{2}{*}{ P-value } & \multirow[b]{2}{*}{ Official gene name } \\
\hline & & Recurrence & No recurrence & & \\
\hline LRRC18 & 1.58 & 21.38 & 13.50 & $1.14^{-04}$ & Leucine rich repeat containing 18 \\
\hline IRAK1BP1 & 1.77 & 90.64 & 51.19 & $6.19^{-04}$ & $\begin{array}{l}\text { Interleukin- } 1 \text { receptor-associated } \\
\text { kinase } 1 \text { binding protein } 1\end{array}$ \\
\hline MGAT4A & 1.57 & 385.69 & 245.89 & $6.00^{-03}$ & $\begin{array}{l}\text { Mannosyl ( } \alpha-1,3-) \text {-glycoprotein } \beta-1 \text {, } \\
\text { 4-N-acetylglucosaminyltransferase, } \\
\text { isozyme A }\end{array}$ \\
\hline BZW2 & 1.74 & 921.80 & 529.93 & $9.33^{-03}$ & $\begin{array}{l}\text { Basic leucine zipper and W2 } \\
\text { domains } 2\end{array}$ \\
\hline LOC 152586 & 1.64 & 19.38 & 11.84 & $1.25^{-02}$ & MGAT4 family, member D \\
\hline ADTRP & 1.78 & 272.08 & 153.19 & $1.46^{-02}$ & $\begin{array}{l}\text { Androgen-dependent TFPI-regulating } \\
\text { protein }\end{array}$ \\
\hline SMC1B & 1.78 & 24.76 & 13.94 & $2.02^{-02}$ & Structural maintenance of chr 1B \\
\hline LOC283788 & 1.53 & 147.03 & 96.39 & $2.39^{-02}$ & Hypothetical protein LOC283788 \\
\hline CLDN12 & 1.52 & 37.24 & 24.58 & $2.56^{-02}$ & Claudin 12 \\
\hline LEF1-AS1 & 1.79 & 74.60 & 41.72 & $3.32^{-02}$ & LEF1 antisense RNA 1 \\
\hline
\end{tabular}

Genes with a fold change $>1.5$ are shown. Data are sorted by P-value. 
Table III. Top 10 downregulated genes.

\begin{tabular}{lcccll}
\hline & & \multicolumn{2}{c}{ Mean of intensity } & & \\
\cline { 3 - 4 } Gene & Fold change & Recurrence & No recurrence & P-value & \multicolumn{1}{c}{ Official gene name } \\
\hline HIST1H2AC & 1.57 & 2086.82 & 3273.74 & $2.50^{-03}$ & Histone cluster 1, H2ac \\
NEXN & 1.65 & 199.36 & 329.40 & $4.46^{-03}$ & Nexilin (F actin binding protein) \\
SIRPB2 & 1.76 & 102.91 & 181.12 & $4.77^{-03}$ & Signal-regulatory protein $\beta 2$ \\
TUBB1 & 1.56 & 462.45 & 719.52 & $5.44^{-03}$ & Tubulin $\beta 1$ \\
HIST1H2BD & 1.56 & 254.17 & 397.28 & $5.81^{-03}$ & Histone cluster 1, H2bd \\
TSPAN2 & 1.76 & 142.35 & 250.81 & $6.02^{-03}$ & Tetraspanin 2 \\
GUCY1A3 & 1.55 & 12.14 & 18.80 & $6.57^{-03}$ & Guanylate cyclase 1, soluble, $\alpha 3$ \\
FAR2 & 1.77 & 129.96 & 230.38 & $7.84^{-03}$ & Fatty acyl CoA reductase 2 \\
STON2 & 1.66 & 91.55 & 151.83 & $9.06^{-03}$ & Nexilin (F actin binding protein) \\
DLEU2 & 1.62 & 63.13 & 102.52 & $1.11^{-02}$ & Deleted in lymphocytic leukemia 2 \\
\hline
\end{tabular}

Genes with a fold change $>1.5$ are shown. Data are sorted by P-value.

$\mathbf{A}$

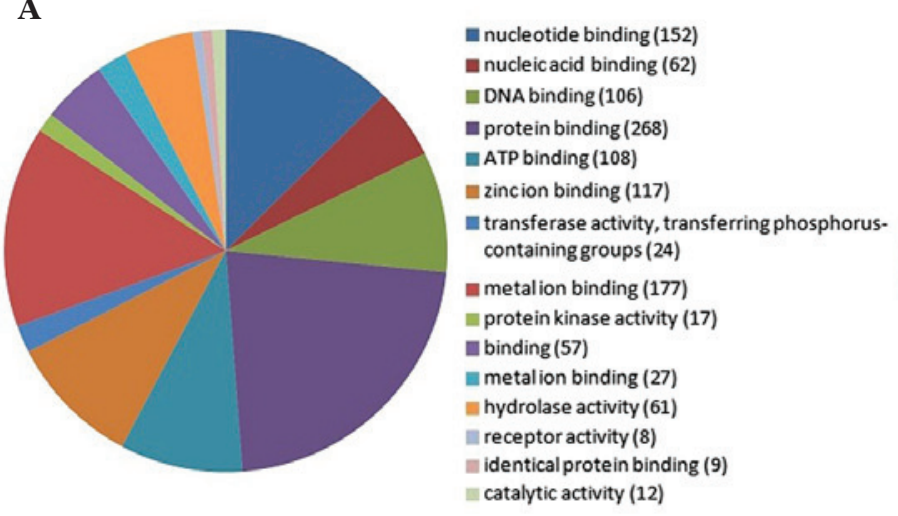

C

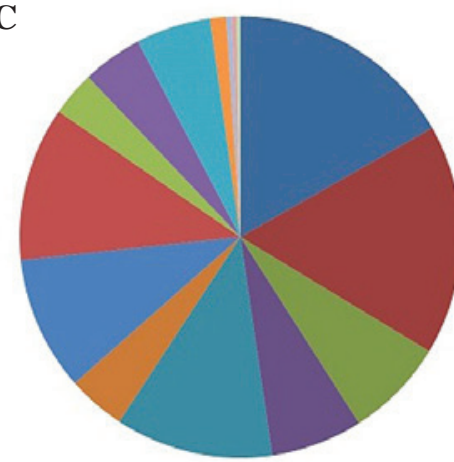

B

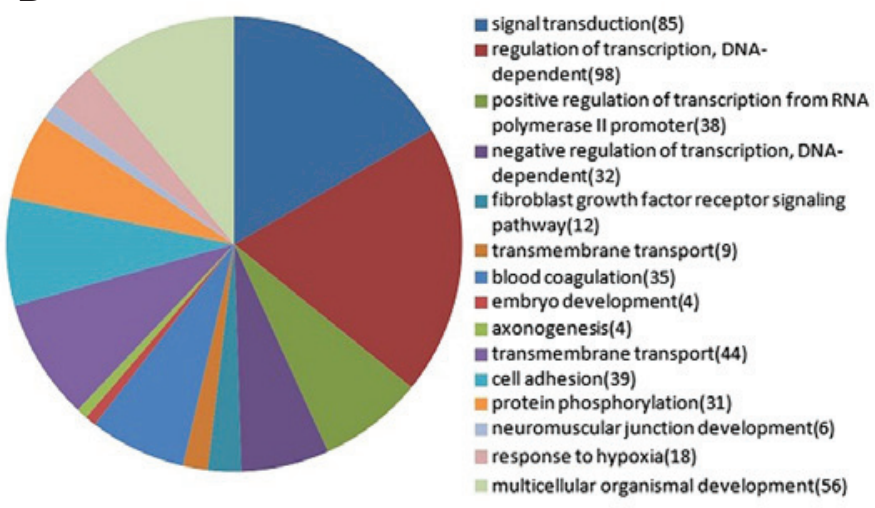

Ecytoplasm (312)

anucleus (314)

incytosol (136)

- intracellular (125)

membrane (215)

Enucleolus (79)

- plasma membrane (183)

- integral to membrane (210)

nucleoplasm (61)

mitochondrion (82)

Extracellular region (102)

inembrane fraction (22)

- Golgi apparatus (7)

Ecytoskeleton (7)

transcription elongation factor complex (5)

Figure 1. GO enrichment. (A) Molecular functions, (B) biological processes and (C) cellular components for the differentially expressed genes. The order of GO enrichment terms were sorted by P-value. GO, gene ontology.

\section{Discussion}

Cardiovascular events are important in the prevention and treatment of cardiovascular diseases. When it occurs in patients with risk factors, the heart function must be re-evaluated, and the prevention and treatment strategy must be adjusted. For patients who had experienced cardiovascular events, the prevention and treatment strategies are not uniform between different regions and hospitals. There are divergences between different area and different grades of hospitals (17), conservative and aggressive strategies are being used, not to mention the circumstances vary among individuals, efficient and effective personalized evaluation and treatment are urged (18). Previous research has revealed that whole-genome sequencing can be used in cardiovascular disease risk-prediction algorithms, to more accurately forecast whether patients will develop 
Table IV. Top 15 enriched KEGG pathways of differentially expressed genes.

\begin{tabular}{|c|c|c|c|}
\hline KEGG ID & KEGG pathway & Gene no. & P-value \\
\hline hsa05200 & Pathways in cancer & 12 & $3.37^{-04}$ \\
\hline hsa05218 & Melanoma & 12 & $3.37^{-04}$ \\
\hline hsa04810 & Regulation of actin cytoskeleton & 9 & $1.65^{-03}$ \\
\hline hsa05215 & Prostate cancer & 11 & $1.77^{-03}$ \\
\hline hsa04010 & MAPK signaling pathway & 23 & $2.04^{-03}$ \\
\hline hsa04120 & Ubiquitin mediated proteolysis & 14 & $6.34^{-03}$ \\
\hline hsa04660 & $\mathrm{T}$ cell receptor signaling pathway & 4 & $7.80^{-03}$ \\
\hline hsa05340 & Primary immunodeficiency & 4 & $7.80^{-03}$ \\
\hline hsa05214 & Glioma & 7 & $8.34^{-03}$ \\
\hline hsa05222 & Small cell lung cancer & 10 & $9.31^{-03}$ \\
\hline hsa04510 & Focal adhesion & 6 & $1.68^{-02}$ \\
\hline hsa04144 & Endocytosis & 16 & $1.98^{-02}$ \\
\hline hsa03030 & DNA replication & 4 & $2.07^{-02}$ \\
\hline hsa03430 & Mismatch repair & 4 & $2.07^{-02}$ \\
\hline hsa04115 & p53 signaling pathway & 8 & $2.28^{-02}$ \\
\hline
\end{tabular}

KEGG, Kyoto Encyclopedia of Genes and Genomes.

disease (19). However, there remains a lack of research about microarray profiling in recurrent cardiovascular events. The present study performed a microarray profiling of peripheral blood samples from patients with AMI, downloaded from the GEO database, to focus on the DEGs of those with or without recurrent cardiovascular events 18 months following AMI.

$\mathrm{R}$ is an integrated suite of software facilities for data manipulation, calculation and graphical display. Using R software and certain packages, the present study identified the DEGs between patients with AMI, with or without recurrent cardiovascular disease. A total of 1,329 genes were identified and 1,023 were upregulated in recurrent group compared with the no recurrent group, while 306 of them were downregulated. The genes with the most significant P-value and fold change $>1.5$ in the up and downregulated DEGs are listed in Tables II and III. Among them, TUBB1 (tubulin $\beta 1$, class VI; $P=0.00544$; fold change $=1.56$ ) encodes a member of the $\beta$ tubulin protein family, and this protein is specifically expressed in platelets and megakaryocytes, and may be involved in proplatelet production and platelet release. Previous research revealed that the prevalence of TUBB1 was higher among healthy individuals compared with patients with cardiovascular disease (20). This may be associated with the TUBB1 function of suppressing microtubule dynamics, fragmenting microtubules and inhibiting cell division (21). Although there is little previous research about other significant genes involved in cardiovascular diseases, the method in the present study may be the initial and alternative way to explore the pathological mechanism of recurrent cardiovascular events.

To further investigate the roles of the DEGs identified in the pathological mechanism of recurrent cardiovascular events, GO enrichment analysis and KEGG pathway analysis was used. GO is widely used as the tool for the organization and functional annotation of molecular aspect (22). It was revealed that the significantly enriched GO terms for molecular functions were nucleotide binding and nucleic acid binding, for biological processes were signal transduction and regulation of transcription (DNA-dependent), and for cellular component were cytoplasm and nucleus. The GO terms mentioned above are basic and vital to the biological and pathological process. Fibroblast growth factor receptor signaling pathway (GO:0008543; $\mathrm{P}=0.00151494$ ), blood coagulation (GO:0007596; $\mathrm{P}=0.00166723$ ) and cell adhesion (GO:0007155; $\mathrm{P}=00170222$ ) were also significantly enriched in GO biological process. Ronca et al (23) reported that fibroblast growth factor receptor-1 gene knockout impairs cardiac and haematopoietic development in murine embryonic stem cells, and the fibroblast growth factor receptor is required for cardiomyocyte differentiation. Yukawa et al (24) demonstrated that impaired fibroblast growth factor receptor gene would suppress the growth of vascular smooth muscle. As for blood coagulation and cell adhesion, which are associated with the formation and breaking off of thrombosis, they are important in both primary and recurrent cardiovascular events.

In KEGG pathway analysis, regulation of actin cytoskeleton is significantly enriched. Actin cytoskeleton is involved in the inward remodeling process associated with cytoskeletal modifications. It is also involved in reducing the passive diameter of resistance vessels, which are the vascular components of the circulatory system, and exert a preponderant role in the regulation of blood flow and the modulation of blood pressure (25). Therefore, the regulation of actin cytoskeleton may have profound consequences on the incidence of cardiovascular events.

The results from PPI network analysis of the top 10 up and downregulated DEGs revealed the significant nodes, including GNG4, MAPK8, PIK3R2, EP300, CREB1 and PIK3CB. MAPK8 is one member of the MAPK family, which has vast implications in signaling and crosstalk with other signaling networks. The MAPK signal pathway is highly associated 


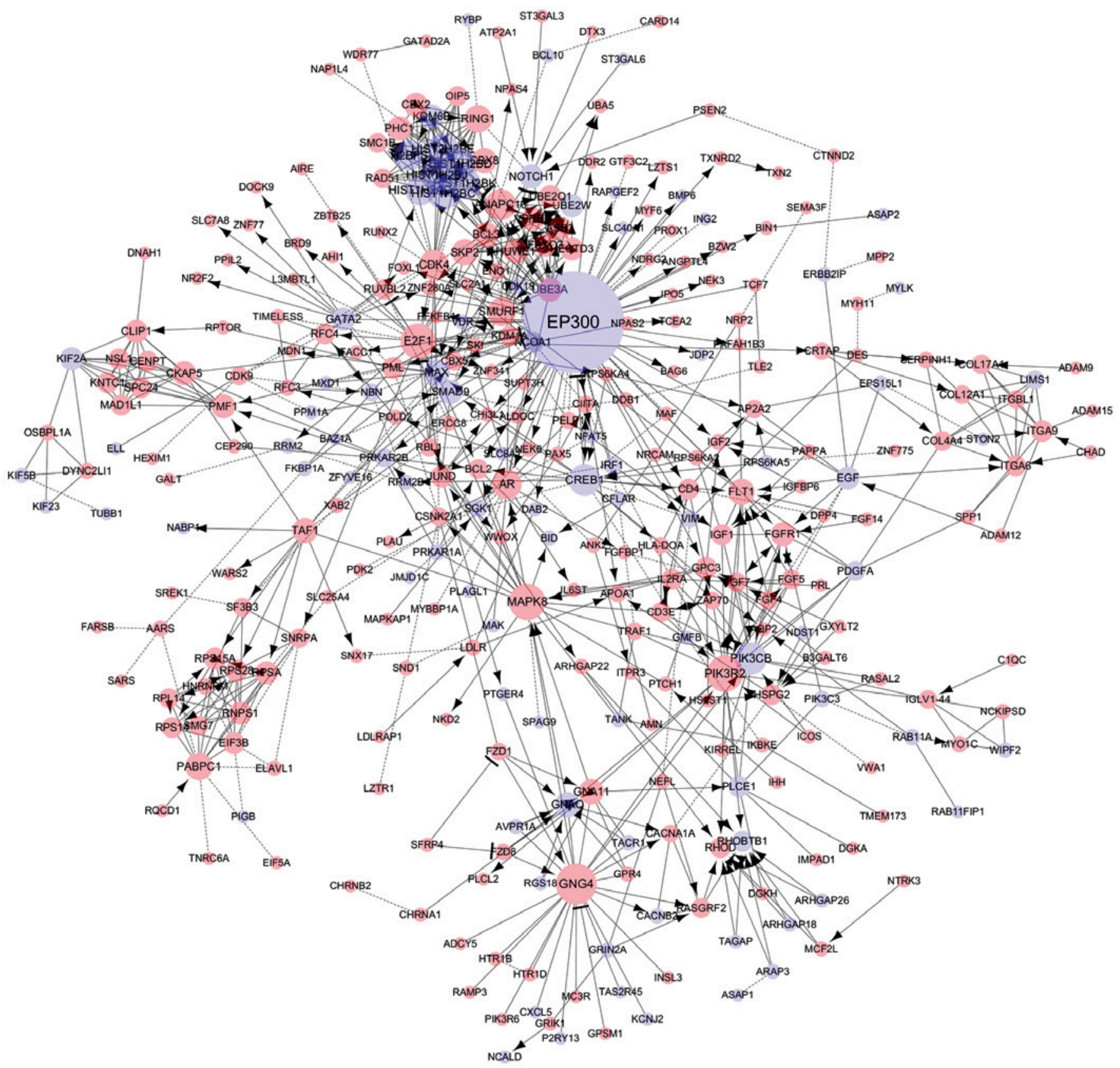

Figure 2. Protein-protein interaction network visualization of differentially expressed genes. The nodes in red represent the upregulated genes and those in blue represent the downregulated genes. The larger size nodes interact more with other nodes. The type of edges represents the interaction between the nodes, $\rightarrow$ to indicate activating/catalyzing, -I to indicate inhibition, - for functional interactions extracted from complexes or inputs and --- for predicted functional interactions.

with mitochondria, the power houses of the cell, which provide $>80 \%$ of ATP for normal cardiomyocyte function and have a crucial role in cell death (26). EP300 is the node with the most interactions with other nodes in the PPI network, and previous research revealed that it is associated with arterial stiffness prior to hypertension, increased pulse pressure, and structural vessel wall changes (27). CREB1, also termed CREB, phosphorylation induced by the prostacyclin/IP pathway may suppress cardiac fibrosis, which is a consequence of numerous cardiovascular diseases, and contributes to impaired ventricular function (28). The PPI results suggested that MAPK8, EP300 and CREB1 may be important in the development of recurrent cardiovascular events.
The results from the present study suggested that DEGs exist between patients with AMI, with and without recurrent cardiovascular events. These genes are involved in different GO enrichment terms and signaling pathways, from which insights into the pathological processes of recurrent events can be obtained. Several genes, including TUBB1, GNG4, MAPK8, PIK3R2, EP300 and CREB1, with or without previous research, may provide potential candidates for distinguishing the susceptibility to recurrent cardiovascular events in the future. Therefore, the present research may provide important references for the prevention and treatment strategies in patients with primary cardiovascular events. Nevertheless, the genes and the associated GO enrichment 


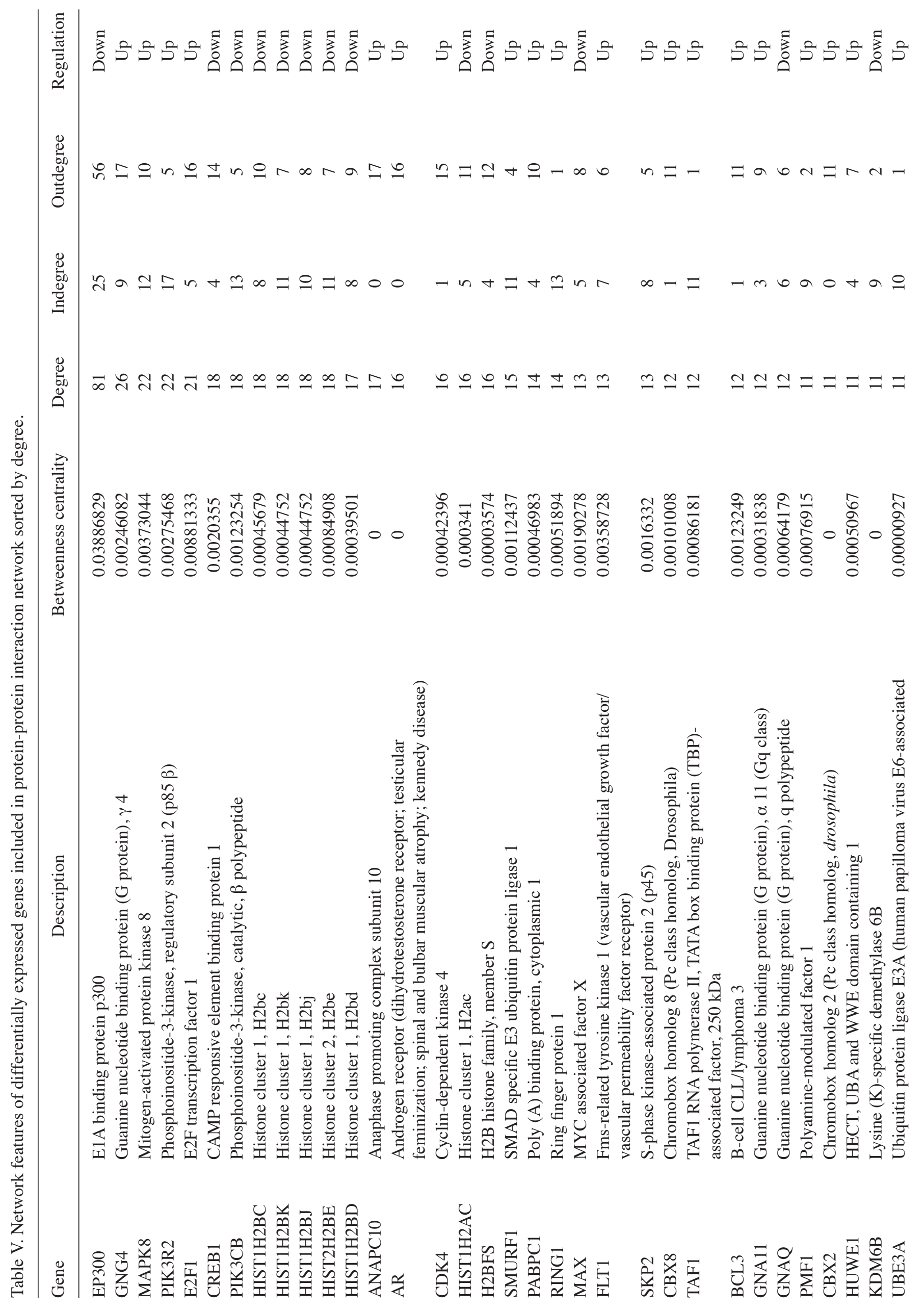




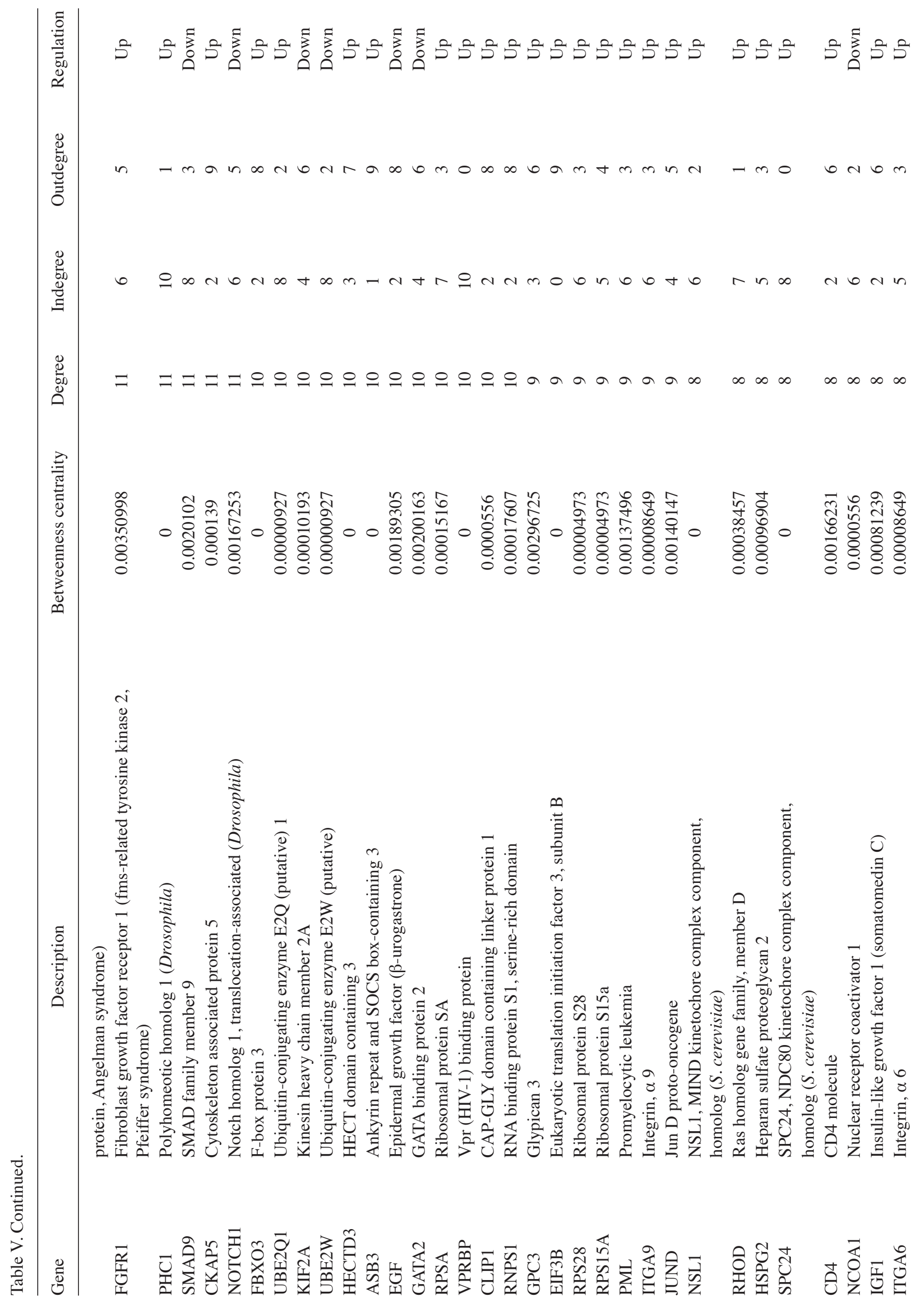




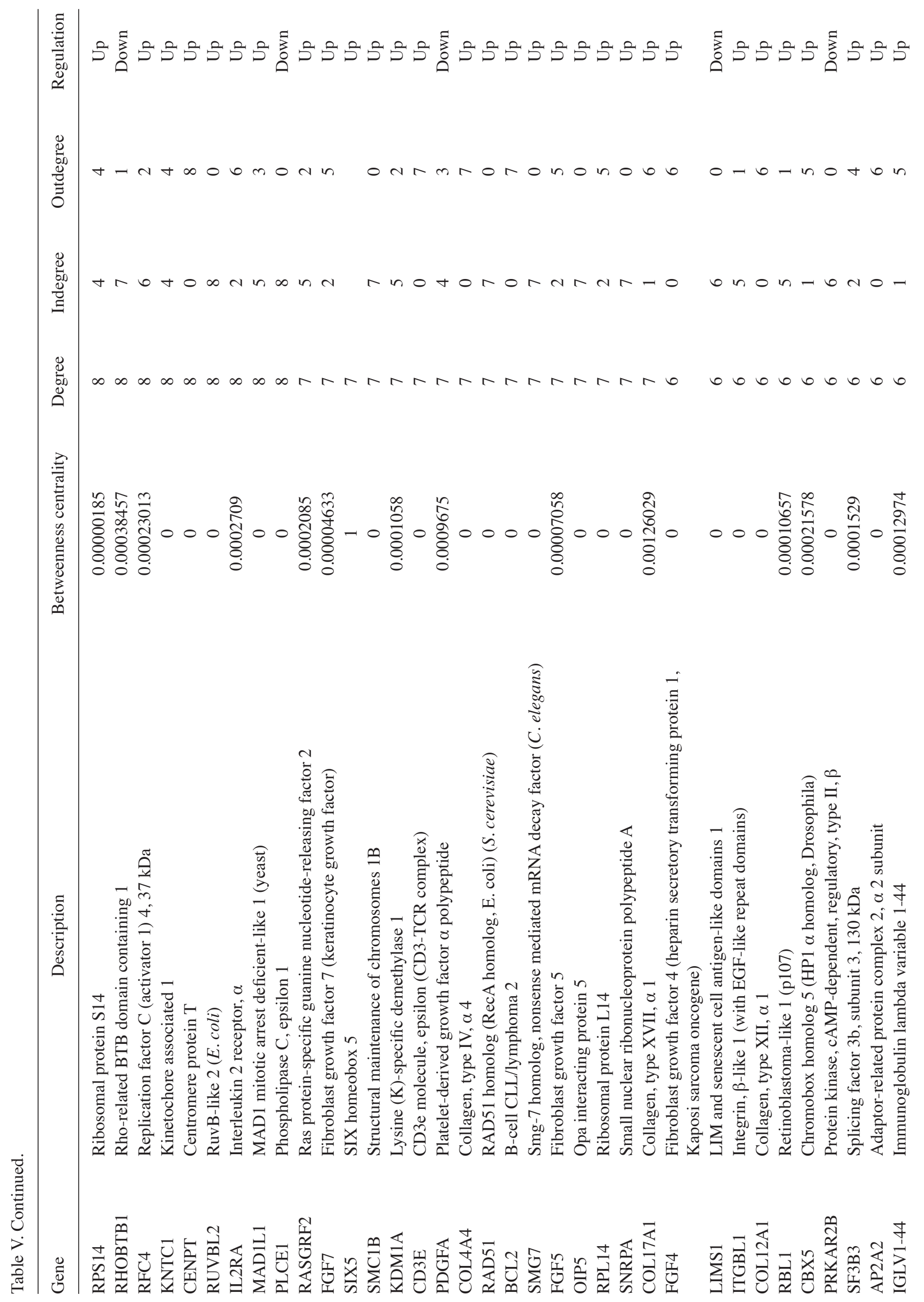




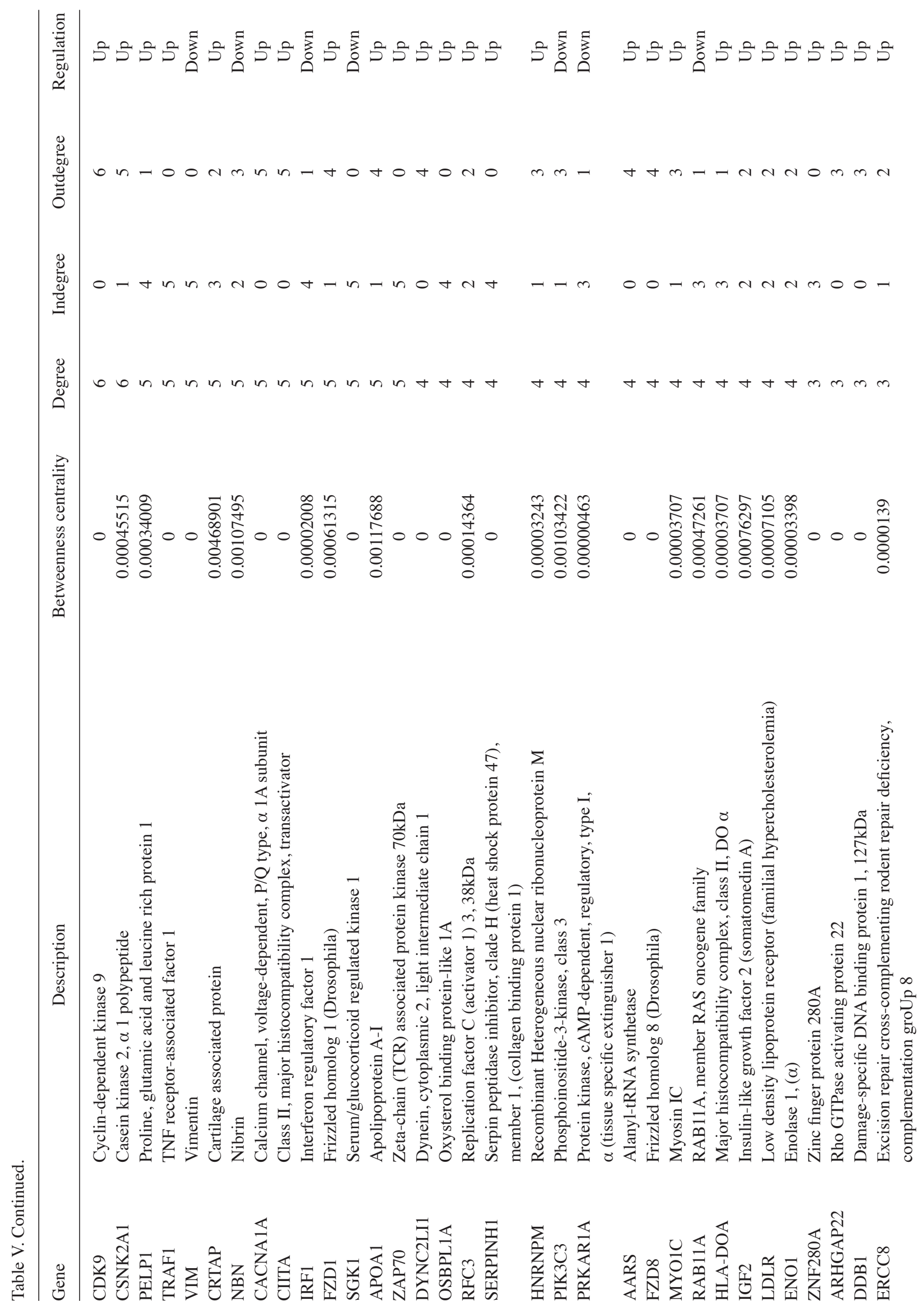




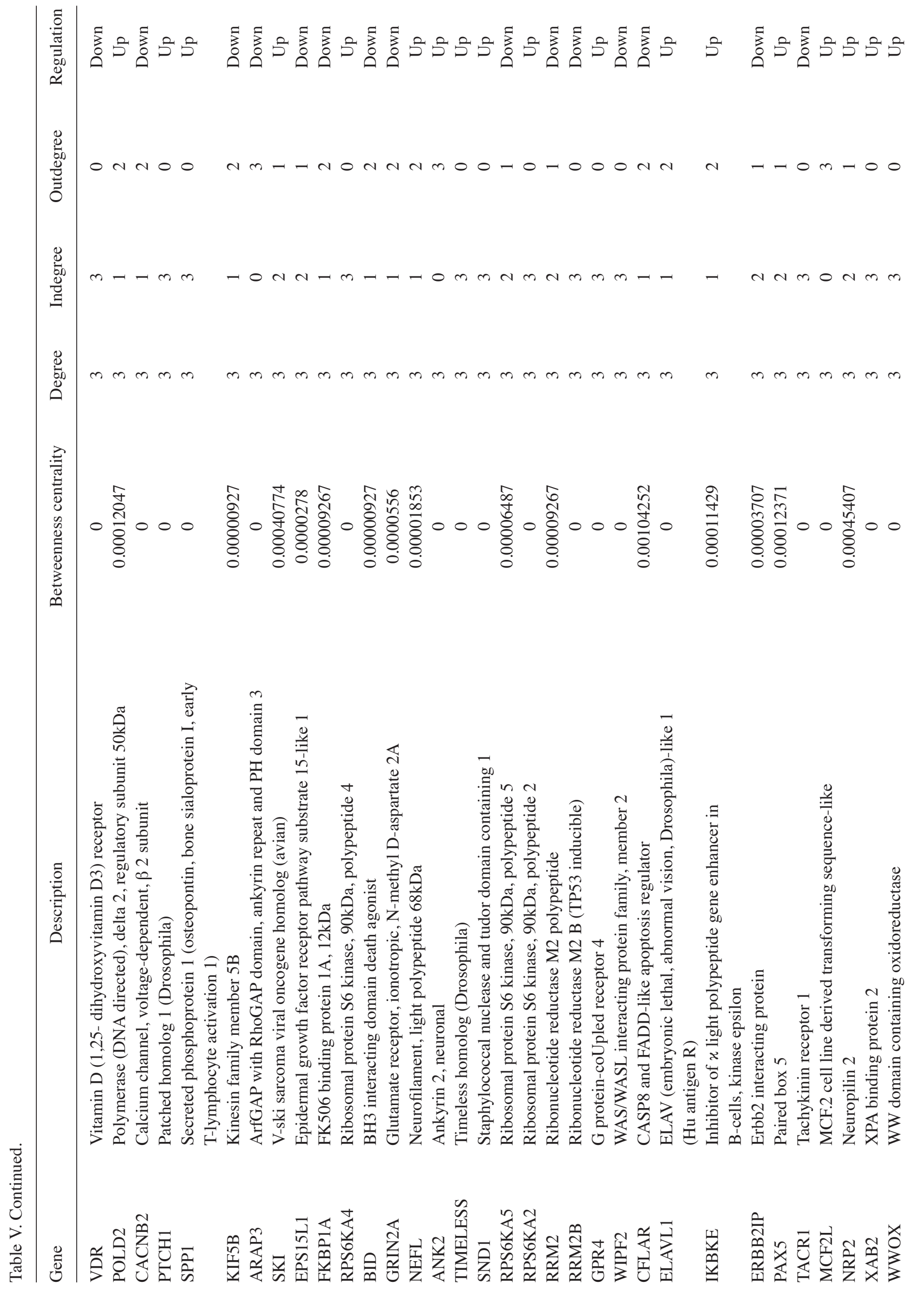




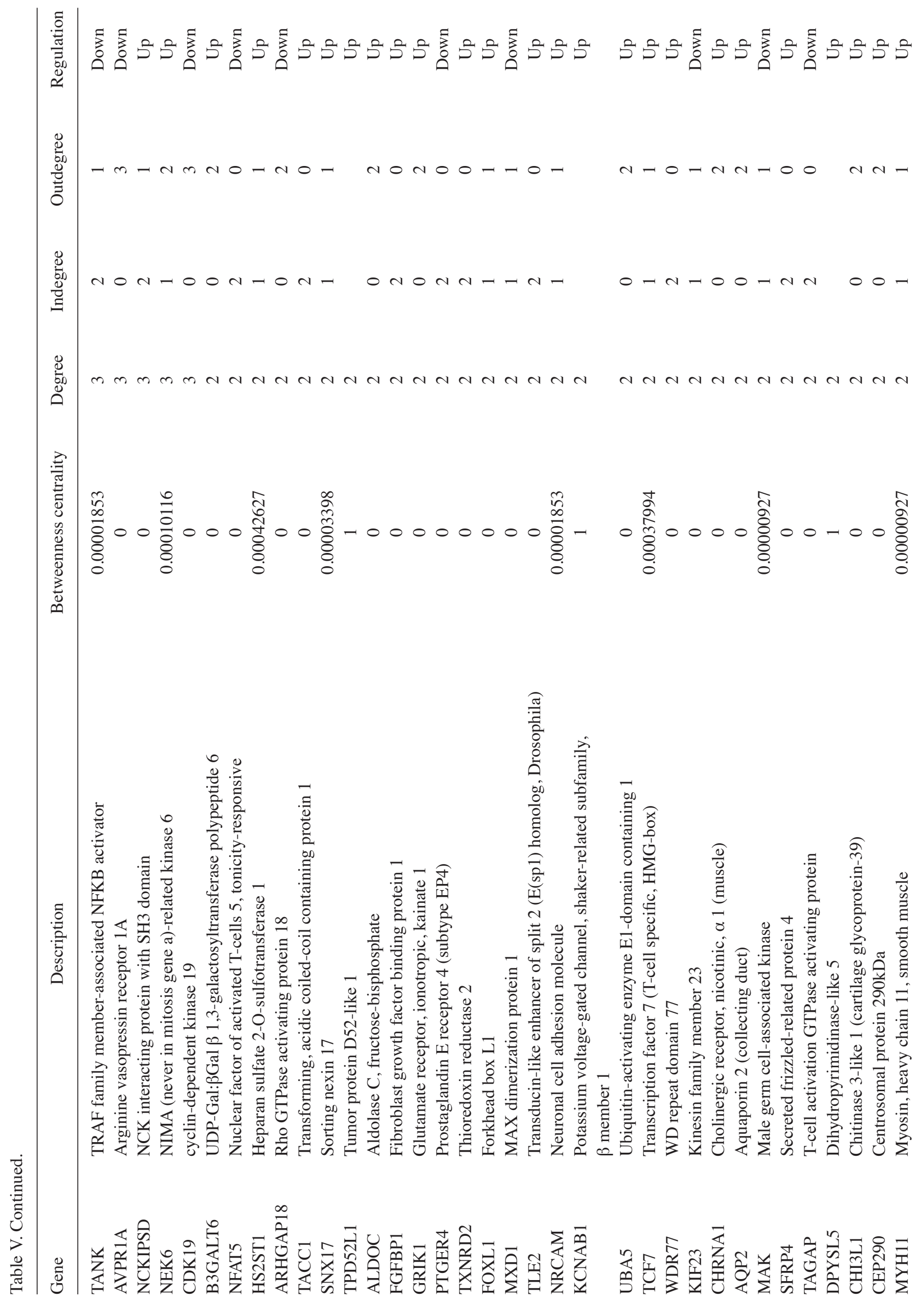




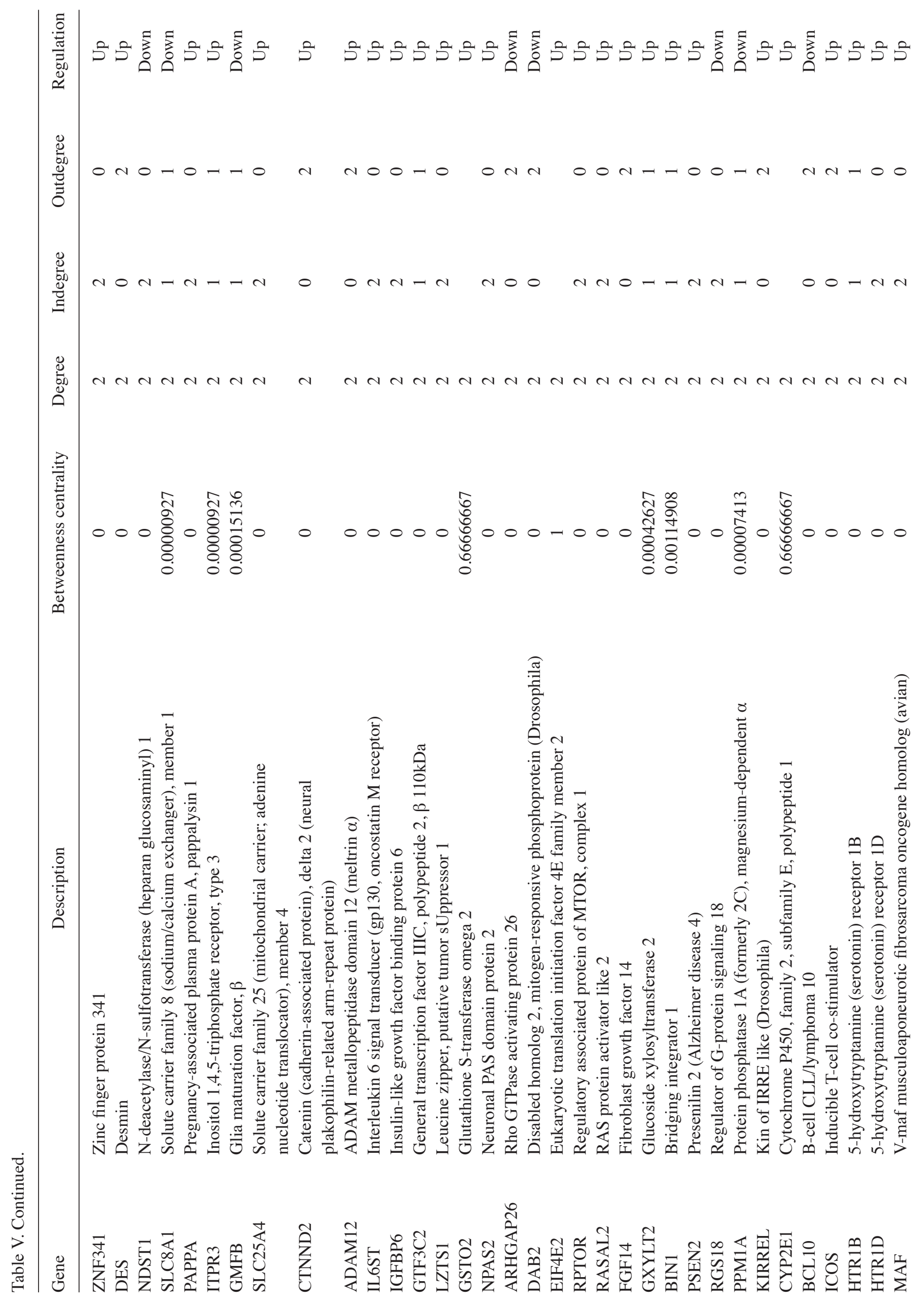




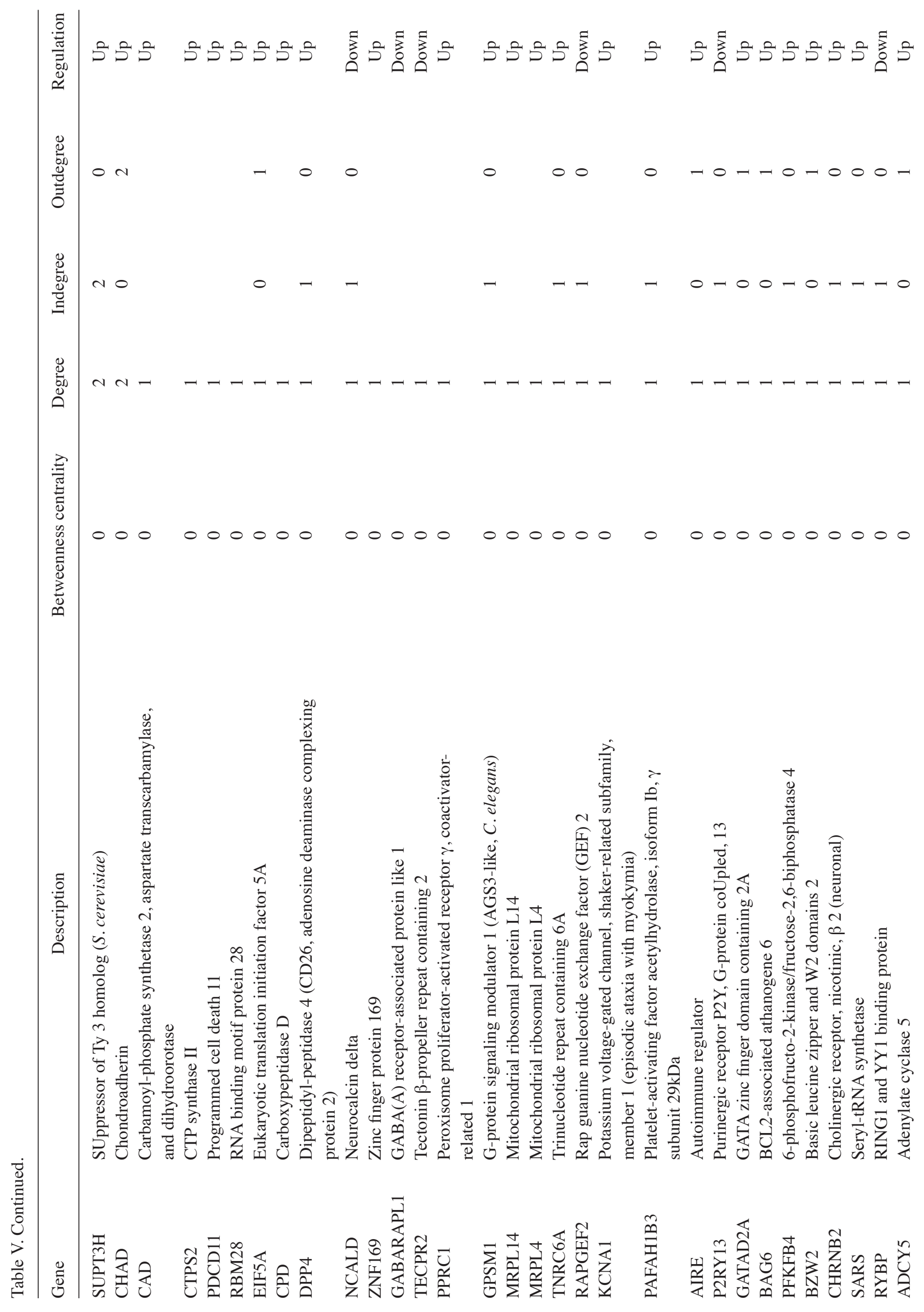




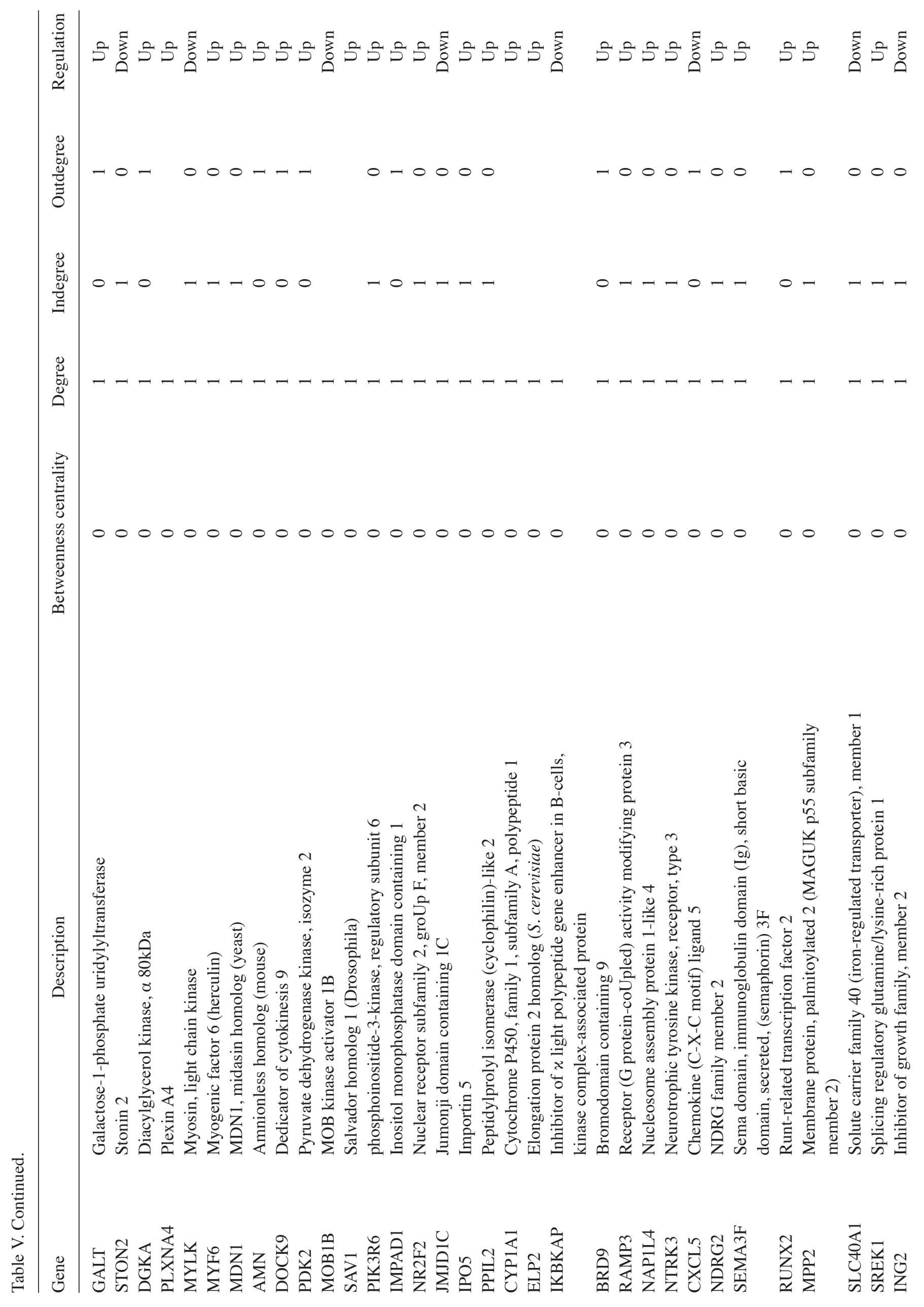




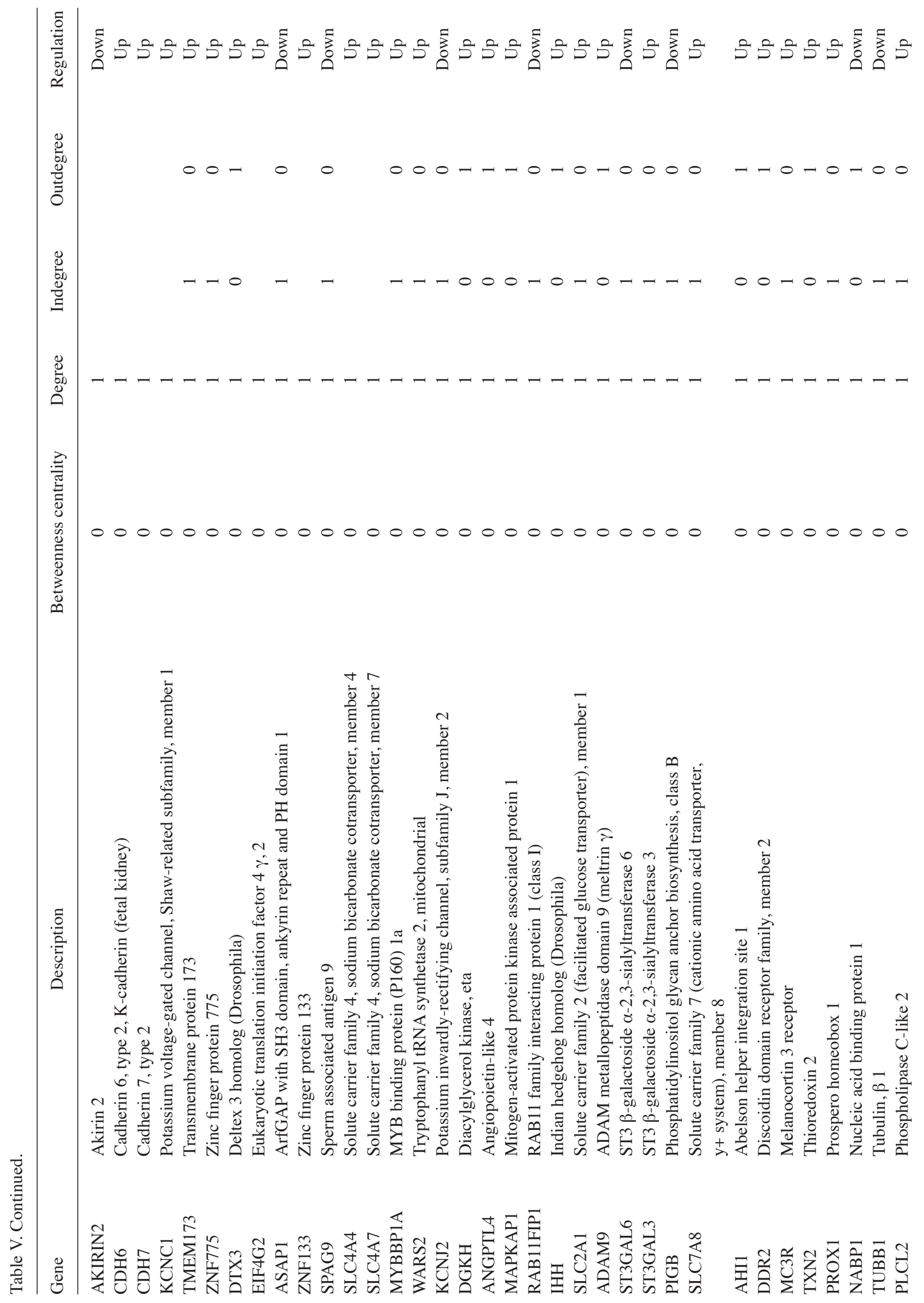




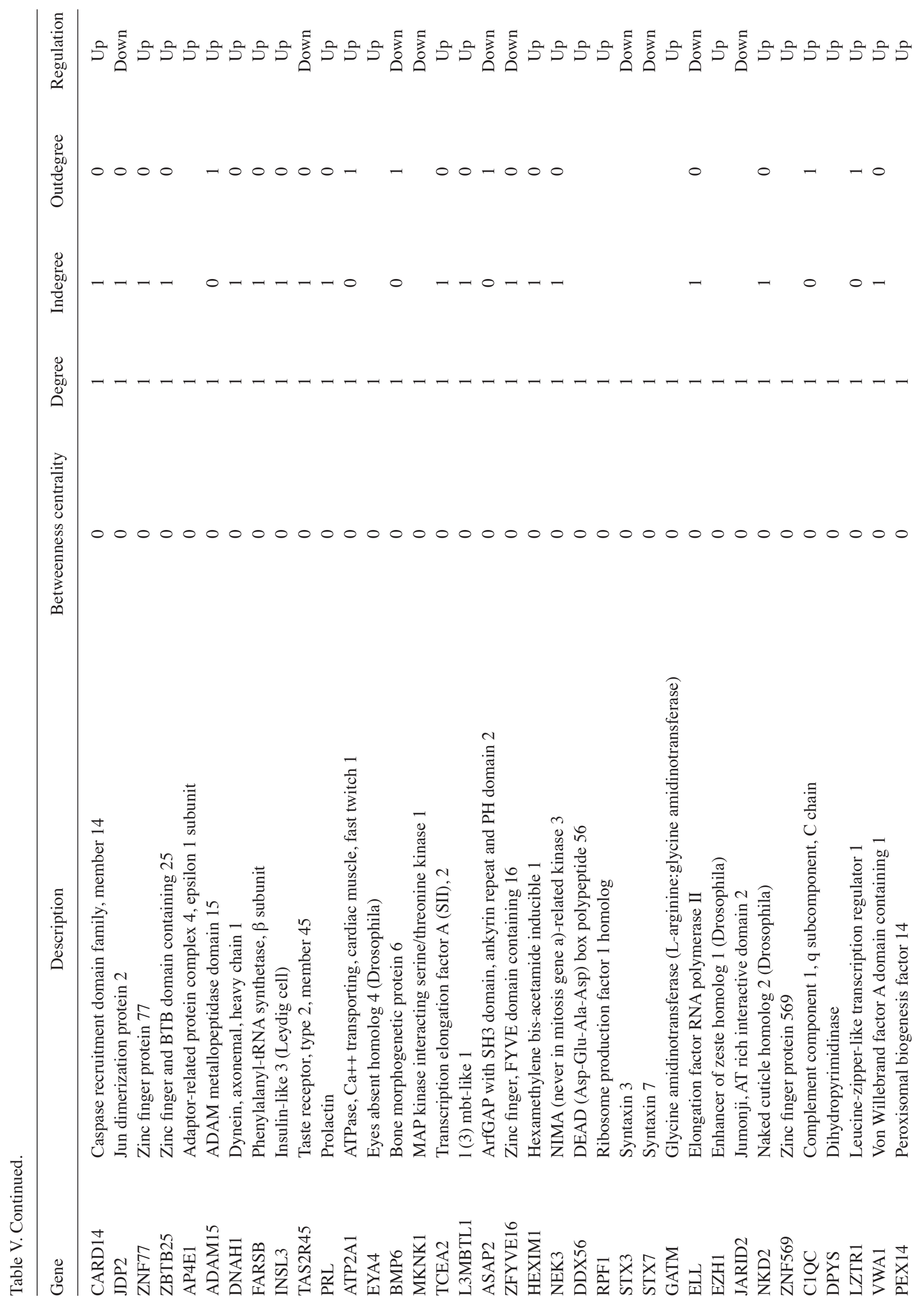




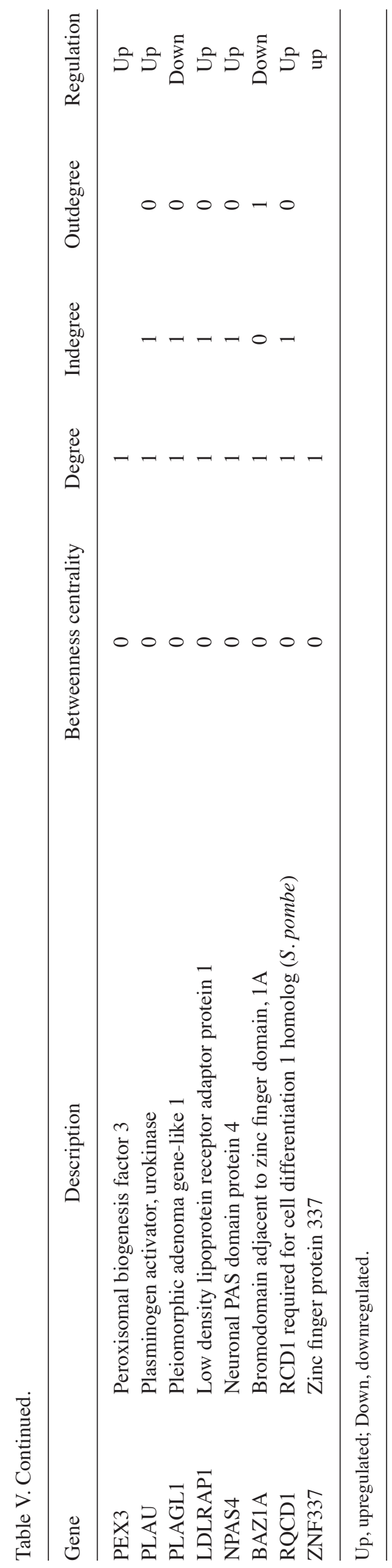


terms and pathways identified here require further investigation and confirmation.

In conclusion, the present study revealed the underlying molecular differences between patients with AMI, with and without recurrent cardiovascular events, including DEGs, their biological function, signaling pathways and key genes in the PPI network, which may contribute to the prevention of recurrent events and personalized treatment for primary cardiovascular events. Further functional studies may provide additional insights into the role of the DEGs in the pathological process of recurrent cardiovascular events.

\section{Acknowledgements}

The present research was supported by a grant from the National Natural Science Foundation of China (no. 81173166).

\section{References}

1. Go AS, Mozaffarian D, Roger VL, Benjamin EJ, Berry JD, Blaha MJ, Dai S, Ford ES, Fox CS, Franco S, et al: Heart disease and stroke statistics-2014 update: A report from the American heart association. Circulation 129: e28-e292, 2014

2. Goff DC Jr, Lloyd-Jones DM, Bennett G, Coady S, D'Agostino RB, Gibbons R, Greenland P, Lackland DT, Levy D, O'Donnell CJ, et al: 2013 ACC/AHA guideline on the assessment of cardiovascular risk: A report of the American college of cardiology/American heart association task force on practice guidelines. Circulation 129 (25 Suppl 2): S49-S73, 2014.

3. Perk J, De Backer G, Gohlke H, Graham I, Reiner Z, Verschuren M, Albus C, Benlian P, Boysen G, Cifkova R, et al: European Guidelines on cardiovascular disease prevention in clinical practice (version 2012). The fifth joint task force of the european society of cardiology and other societies on cardiovascular disease prevention in clinical practice (constituted by representatives of nine societies and by invited experts). Eur Heart J 33: 1635-1701, 2012.

4. Ceška R and Stulc T: Implementation of cardiovascular disease prevention guidelines into clinical practice: An unmet challenge? Curr Pharm Des 21: 1180-1184, 2015.

5. van Staa TP, Gulliford M, Ng ES, Goldacre B and Smeeth L: Prediction of cardiovascular risk using framingham, ASSIGN and QRISK2: How well do they predict individual rather than population risk? PLoS One 9: e106455, 2014.

6. Taljaard M, Tuna M, Bennett C, Perez R, Rosella L, Tu JV, Sanmartin C, Hennessy D, Tanuseputro P, Lebenbaum M and Manuel DG: Cardiovascular disease population risk tool (CVDPoRT): Predictive algorithm for assessing CVD risk in the community setting. A study protocol. BMJ Open 10: e006701, 2014.

7. Cui J, Forbes A, Kirby A, Marschner I, Simes J, Hunt D, West M and Tonkin A: Semi-parametric risk prediction models for recurrent cardiovascular events in the LIPID study. BMC Med Res Methodol 10: 27, 2010.

8. Xu F, Teng X, Yuan X, Sun J, Wu H, Zheng Z, Tang Y and Hu S: LCK: A new biomarker candidate for the early diagnosis of acute myocardial infarction. Mol Biol Rep 41: 8047-8053, 2014.

9. Duan L, Xiong X, Liu Y and Wang J: miRNA-1: Functional roles and dysregulation in heart disease. Mol Biosyst 10: 2775-2782, 2014.

10. Tikkanen E, Havulinna AS, Palotie A, Salomaa V and Ripatti S: Genetic risk prediction and a 2 -stage risk screening strategy for coronary heart disease. Arterioscler Thromb Vasc Biol 33: 2261-2266, 2013
11. Suresh R, Li X, Chiriac A, Goel K, Terzic A, Perez-Terzic C and Nelson TJ: Transcriptome from circulating cells suggests dysregulated pathways associated with long-term recurrent events following first-time myocardial infarction. J Mol Cell Cardiol 74: 13-21, 2014.

12. Gautier L, Cope L, Bolstad BM and Irizarry RA: Affy-analysis of Affymetrix GeneChip data at the probe level. Bioinformatics 20: 307-315, 2004.

13. Smyth GK, Michaud J and Scott HS: Use of within-array replicate spots for assessing differential expression in microarray experiments. Bioinformatics 21: 2067-2075, 2005.

14. Nogales-Cadenas R, Carmona-Saez P, Vazquez M, Vicente C, Yang X, Tirado F, Carazo JM and Pascual-Montano A: GeneCodis: Interpreting gene lists through enrichment analysis and integration of diverse biological information. Nucleic Acids Res 37: W317-W322, 2009.

15. Carmona-Saez P, Chagoyen M, Tirado F, Carazo JM and Pascual-Montano A: GENECODIS: A web-based tool for finding significant concurrent annotations in gene lists. Genome Biol 8: $\mathrm{R} 3,2007$.

16. Saito R, Smoot ME, Ono K, Ruscheinski J, Wang PL, Lotia S, Pico AR, Bader GD and Ideker T: A travel guide to cytoscape plugins. Nat Methods 9: 1069-1076, 2012.

17. Mortensen MB and Falk E: Real-life evaluation of European and American high-risk strategies for primary prevention of cardiovascular disease in patients with first myocardial infarction. BMJ Open 4: e005991, 2014.

18. Montecucco F, Carbone F, Dini FL, Fiuza M, Pinto FJ, Martelli A, Palombo D, Sambuceti G, Mach F and De Caterina R: Implementation strategies of systems medicine in clinical research and home care for cardiovascular disease patients. Eur J Intern Med 25: 785-794, 2014.

19. Musunuru K: Personalized genomes and cardiovascular disease. Cold Spring Harb Perspect Med 5: a014068, 2014.

20. Freson K, De Vos R, Wittevrongel C, Thys C, Defoor J, Vanhees L, Vermylen J, Peerlinck K and Van Geet C: The TUBB1 Q43P functional polymorphism reduces the risk of cardiovascular disease in men by modulating platelet function and structure. Blood 106: 2356-2362, 2005.

21. Yang H, Ganguly A, Yin S and Cabral F: Megakaryocyte lineage-specific class VI $\beta$-tubulin suppresses microtubule dynamics, fragments microtubules, and blocks cell division. Cytoskeleton (Hoboken) 68: 175-187, 2011.

22. Lovering RC, Camon EB, Blake JA and Diehl AD: Access to immunology through the gene ontology. Immunology 125: 154-160, 2008

23. Ronca R, Gualandi L, Crescini E, Calza S, Presta M and Dell'Era P: Fibroblast growth factor receptor-1 phosphorylation requirement for cardiomyocyte differentiation in murine embryonic stem cells. J Cell Mol Med 13: 1489-1498, 2009.

24. Yukawa H, Miyatake SI, Saiki M, Takahashi JC, Mima T, Ueno H, Nagata I, Kikuchi H and Hashimoto N: In vitro growth suppression of vascular smooth muscle cells using adenovirus-mediated gene transfer of a truncated form of fibroblast growth factor receptor. Atherosclerosis 141: 125-132, 1998.

25. Castorena-Gonzalez JA, Staiculescu MC, Foote $\mathrm{C}$ and Martinez-Lemus LA: Mechanisms of the inward remodeling process in resistance vessels: Is the actin cytoskeleton involved? Microcirculation 21: 219-229, 2014.

26. Javadov S, Jang S and Agostini B: Crosstalk between mitogen-activated protein kinases and mitochondria in cardiac diseases: Therapeutic perspectives. Pharmacol Ther 144: 202-225, 2014.

27. Herrera VL, Decano JL, Giordano N, Moran AM and Ruiz-Opazo N: Aortic and carotid arterial stiffness and epigenetic regulator gene expression changes precede blood pressure rise in stroke-prone Dahl salt-sensitive hypertensive rats. PLoS One 9: e107888, 2014.

28. Chan EC, Dusting GJ, Guo N, Peshavariya HM, Taylor CJ, Dilley R, Narumiya S and Jiang F: Prostacyclin receptor suppresses cardiac fibrosis: Role of CREB phosphorylation. J Mol Cell Cardiol 49: 176-185, 2010. 